\title{
Triple arterial blood supply to the liver and double cystic arteries
}

\author{
J. Dolenšek \\ Institute of Anatomy, Faculty of Medicine, University of Ljubljana, Slovenia
}

[Received: 29 November 2016; Accepted: 2 January 2017]

\begin{abstract}
A rare combination of variations in the arterial supply of the liver and gallbladder was encountered in a male cadaver. The simultaneous occurrence of an accessory left hepatic artery and an accessory right hepatic artery from which double cystic arteries arose (one of which was low-lying). This combination has not yet been reported. The accessory left hepatic artery originated from the left gastric artery. The accessory right hepatic artery originated from the superior mesenteric artery. Such arterial variations are caused by differences in embryological development. This, however, may lead to complications related to diagnostic and therapeutic procedures in the upper abdomen. (Folia Morphol 2017; 76, 3: 523-526)
\end{abstract}

Key words: hepatic arteries variations, accessory hepatic artery, low-lying cystic artery

\section{INTRODUCTION}

Variations in the arterial blood supply to the liver and gallbladder are common. The typical textbook pattern, in which the liver receives its total arterial supply from the hepatic branch of the coeliac trunk (CT), occurs in $55-88 \%$ of cases $[1,7,8]$. Under variant patterns, however, the hepatic lobes may receive arterial blood from the left gastric artery (LGA), the superior mesenteric artery (SMA), the aorta or other visceral arteries $[6-8,11,13]$. These aberrant arteries may be either accessory in nature, occurring in addition to the normal arterial supply, or replaced, thereby substituting the normal arterial supply to the lobe $[6,7,13]$.

The typical pattern of the blood supply to the gallbladder by a single cystic artery (CA), arising from the right hepatic artery (RHA) in the hepatobiliary triangle, occurs in $63 \%$ of cases [8]. The hepatobiliary triangle is the area bounded by the cystic duct, the common hepatic duct and the inferior border of the liver [2]. The CA typically traverses the hepatobiliary triangle to the right of the common hepatic duct towards the neck of the gallbladder, where it normally divides into superficial and deep branches. The superficial branch supplies the free peritoneal surface of the gallbladder. The deep branch runs in the gallbladder fossa, between the liver and the gallbladder [8]. Variant CA anatomy includes variations in its origin, course and number of vessels $[2,5,10,11]$.

\section{CASE REPORT}

During a routine dissection of an adult male cadaver, a triple arterial blood supply to the liver and double cystic arteries to the gallbladder were found (Fig. 1). The CT trifurcated classically with the LGA branching out first, followed by the splenic and common hepatic branches. The LGA supplied the lesser curvature of the stomach with a few branches. The accessory left hepatic artery (aLHA) ran upwards in the hepatogastric ligament as a continuation of the

Address for correspondence: Dr. J. Dolenšek, Institute of Anatomy, Faculty of Medicine, Korytkova 2, 1000 Ljubljana, Slovenia, tel: +386-1-5437303, fax: +386-1-5437301, e-mail: janez.dolensek@gmail.com; janez.dolensek@mf.uni-lj.si 


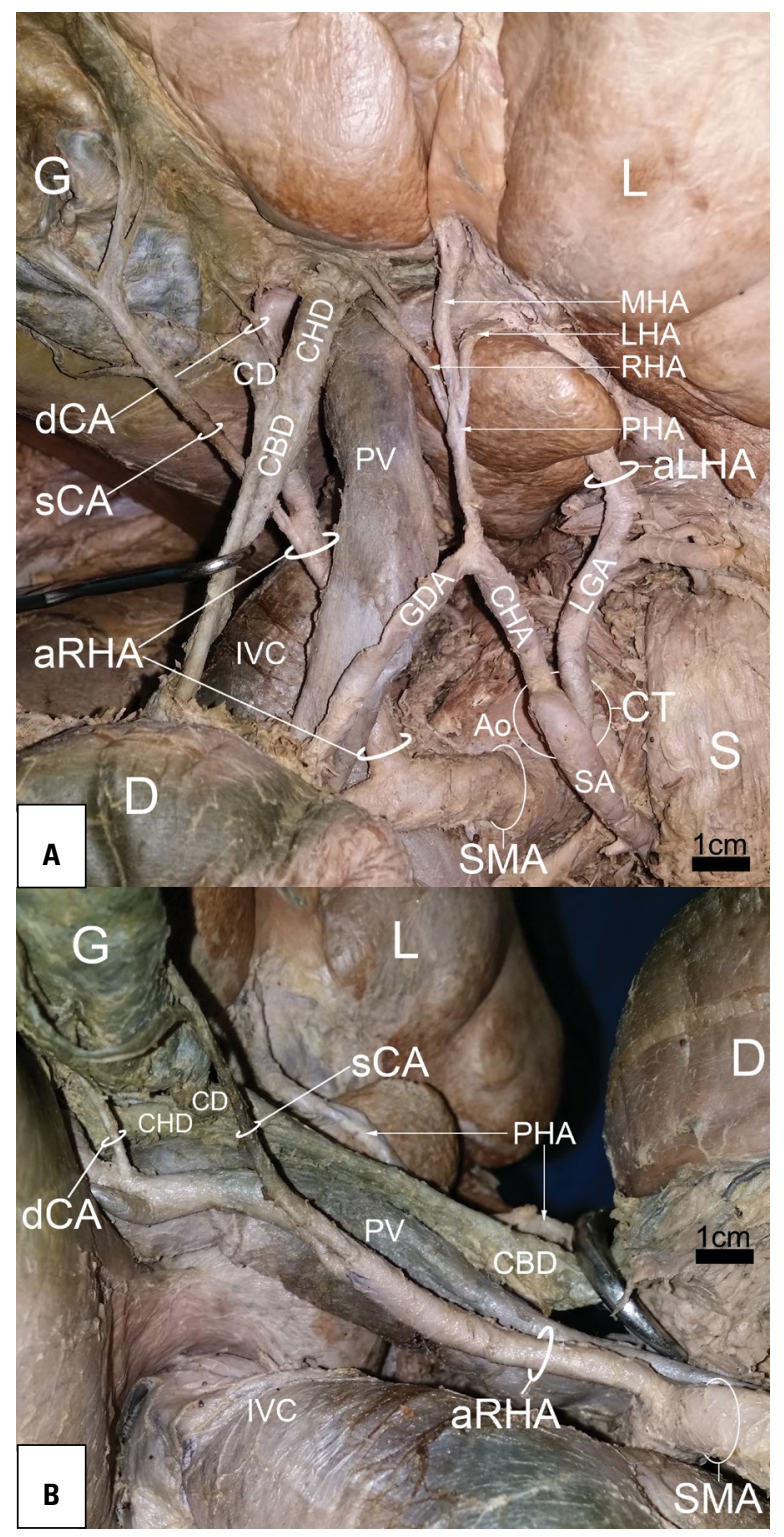

Figure 1. Triple arterial blood supply to the liver and double cystic arteries to the gallbladder, A. Anterior view; B. Right lateral view; aLHA — accessory left hepatic artery; aRHA — accessory right hepatic artery; SCA - superficial cystic artery; $\mathrm{dCA}$ - deep cystic artery; $\mathrm{CD}$ - cystic duct; $\mathrm{CBD}$ - common bile duct; $\mathrm{CHD}$ common hepatic duct; CT — celiac trunk; LGA — left gastric artery; SA — splenic artery; CHA — common hepatic artery; GDA gastroduodenal artery; PHA — proper hepatic artery; MHA — middle hepatic artery; LHA — left hepatic artery; RHA — right hepatic artery; SMA — superior mesenteric artery; Ao — aorta; IVC inferior vena cava; PV — portal vein; $\mathrm{G}$ — gallbladder; $\mathrm{L}$ — liver; $\mathrm{D}$ - duodenum; S - stomach.

LGA before turning around a prominent papillary process of the caudate lobe and entering into the hepatic porta. The common hepatic artery $(\mathrm{CHA})$ gave rise to the gastroduodenal artery (GDA) and the right gastric artery, as expected, before continuing as the proper hepatic artery (PHA). The PHA trifurcated into the middle hepatic artery (MHA) and the narrower left and right hepatic arteries.

The accessory right hepatic artery (aRHA) derived from the SMA passed obliquely upward, posterior to the common bile duct in the hepatoduodenal ligament before branching off into double cystic arteries: the superficial cystic artery ( $\mathrm{SCA}$ ) on its half way to the liver and the deep cystic artery (dCA) in the hepatobiliary triangle. The larger sCA, $3 \mathrm{~mm}$ in diameter, was unusually long $(50 \mathrm{~mm})$, low-lying and ran beyond the hepatobiliary triangle through the cholecystoduodenal ligament, on the surface of the gallbladder's body. In addition to this, a miniature dCA, only $1.8 \mathrm{~mm}$ in diameter, ran through the middle of the hepatobiliary triangle towards the gallbladder's fossa.

The diameters of the three arteries supplying the liver were: the PHA $(3 \mathrm{~mm})$, the aLHA $(5 \mathrm{~mm})$ and the aRHA $(6 \mathrm{~mm})$.

\section{DISCUSSION}

Knowledge of embryology is vital in order to understand the formation of arterial variations in the liver. Mammalian liver development starts with three embryonic lobes - one central and two lateral - which are closely connected with the embryonic (umbilical, vitelline) veins $[3,4]$. Each embryonic lobe is supplied by its own embryonic artery (EA): the left lateral lobe is supplied by the left $E A$, originating from LGA; the central lobe is supplied by the middle EA from the $C T$; and the right lateral lobe is supplied by the right EA from the SMA. The three aforementioned embryonic arteries (left, middle and right) anastomose in the hepatic hilum. The central lobe, which later develops in right and left paramedian sectors, grows intensively and overlaps the lateral lobes (future lateral sectors). Lastly, owing to the enlargement of the stomach and spleen, the left lateral embryonic lobe grows less than the right. Consequently, the shape of the liver becomes remarkably asymmetric, as is the case in a fully developed liver [4]. With the hepatic growth, the middle EA territory continues to enlarge until it becomes a dominant hepatic artery. The middle EA then develops into the PHA, with a left and right branch (i.e. normal arterial liver supply). The left and right embryonic arteries gradually regress to the point where they finally disappear. Only minuscule fibrous remnants may be found.

In human foetuses in the 4th month of pregnancy, a left hepatic EA was found in $50 \%$ of cases 
and a right hepatic EA was found in $25 \%$ [4]. Miyaki (1989) [9] analysed 60 foetal livers over 5 gestational months old and found a left hepatic EA in $25.0 \%$ and right hepatic EA in $18.3 \%$ of cases. The left and right hepatic embryonic arteries coexisted with a regular arterial liver supply in $10.0 \%$ of cases. Therefore, the occurrence of hepatic EA progressively decreases with increasing gestational age. In the adult population, the simultaneous occurrence of the aLHA and aRHA was observed in addition to the ordinary arterial liver supply in only $1-3 \%$ of cases $[1,8]$. It can therefore be hypothesized that the aLHA and aRHA are remainders of the left and right hepatic embryonic arteries.

From a functional standpoint, it may be concluded that in the presented case (Fig. 1) each of the accessory hepatic arteries (aLHA, aRHA) with diameters of $5 \mathrm{~mm}$ and $6 \mathrm{~mm}$, respectively, probably provided more blood flow to the liver than the PHA, the diameter of which was only $3 \mathrm{~mm}$. Regarding the arterial supply of the gallbladder, the sCA clearly dominated. This can be confirmed from the SCA dimensions as well as from the distribution pattern of its sub-branches. Some of these sub-branches were actually larger than the dCA itself and even reached the fundus of the gallbladder.

Thorough knowledge of anatomical variations serves as the foundation for any hepatobiliary surgical procedure, including transplantation. Due to a lack of donor livers, each organ is precious, regardless of its arterial supply variation. In the presented case (Fig. 1), the liver is supplied by both the CT (via the CHA and aLHA) and the SMA (via the aRHA). All accessory arteries must be preserved during transplantation because all hepatic arteries are terminal vessels which supply specific territories of the liver [8]. In a whole liver (orthotopic) transplantation, the arterial blood supply to a hepatic graft is usually provided by an end-to-end anastomosis between the donor's $\mathrm{CT}$ and the recipient's CHA [12]. If the donor's liver is supplied by more than one artery, as in the case presented, the donor's variant arterial liver supply must be converted into a single vessel during the transplantation procedure. Arterial supply to the left lateral hepatic sector from the LGA (via aLHA) presents no problem because the LGA arises from the CT. The partial or entire right lobe arterial supply by the aRHA from the SMA represents a serious surgical problem with various possible solutions [12].

Variant CA anatomy is also common. The double cystic arteries occurring in $12-25 \%$ and represent cases in which the superficial and deep branches of the CA have separate origins, either from the same source or two different sources $[5,8]$. Both cystic branches most commonly originate from the RHA [10]. It is slightly less common for the dCA to arise from the RHA and the SCA to arise from the GDA or some other source. Sometimes both cystic arteries stem from an anomalous source [8]. If one of the cystic arteries is located within the hepatobiliary triangle and the other is located outside, as in the case presented, this represents a compound CA type [5]. In patients with double cystic arteries, both must be found and ligated during the cholecystectomy.

When the CA arises either from the GDA and its branches or from the SMA, it often ascends to the gallbladder early, forming a low-lying CA configuration. This artery approaches the gallbladder inferior to the cystic duct and never enters into the hepatobiliary triangle [2]. A low-lying CA may provide the gallbladder with complete arterial supply or, as in the case presented, it simply represents a superficial branch of the double cystic arteries (Fig. 1). A lowlying CA is clinically important because a surgeon ordinarily does not expect to observe the CA inferior to the cystic duct. From a laparoscopic perspective, it is located more superficially and anterior to the cystic duct, which means that it is the first structure encountered during a cholecystectomy. If a low-lying CA is overlooked, this may lead to an accidental intersection with an unintended haemorrhage [2]. Variant CA anatomy is difficult to establish before a cholecystectomy and so careful dissection of the hepatobiliary triangle and the gallbladder must be performed in order to avoid unnecessary complications. Bleeding from the CA can be a cause for a conversion from a laparoscopic to an open cholecystectomy [5].

Cases with one accessory hepatic artery associated with double cystic arteries are very rare [11]. Simultaneous occurrence of aLHA, aRHA and double cystic arteries arising from the latter, is a feature that has, to the best of our knowledge, not been reported to date.

\section{CONCLUSIONS}

Anatomical variations of this type could be the result of some persistence in the foetal arterial hepatic pattern. Adequate familiarity with the variants of the anatomy of cystic and hepatic arteries is a precondition for performing accurate diagnostic and safe surgical procedures in the upper abdomen. 


\section{Acknowledgements}

We are grateful to Friderik Štendler for his help with the dissection.

\section{REFERENCES}

1. Adachi B. Das Arteriensystem der Japaner. Band II. Verlag Der Kaiserlich-Japanischen Universität zu Kyoto, Kyoto 1928.

2. Balija M, Huis M, Nikolic V, et al. Laparoscopic visualization of the cystic artery anatomy. World J Surg. 1999; 23(7): 703-7; discussion 707, indexed in Pubmed: 10390590.

3. Bradley OC. A contribution to the morphology and development of the mammalian liver. J Anat Physiol. 1908; 43: 1-42, indexed in Pubmed: 17232788.

4. Couinaud C. Surgical anatomy of the liver revisited. Embryology, Paris 1989: 11-24.

5. Ding YM, Wang B, Wang WX, et al. New classification of the anatomic variations of cystic artery during laparoscopic cholecystectomy. World J Gastroenterol. 2007; 13(42): 5629-5634, indexed in Pubmed: 17948938.

6. Gurgacz AM, Horbaczewska A, Klimek-Piotrowska W, et al. Variations in hepatic vascularisation: lack of a proper hepatic artery. Two case reports. Folia Morphol. 2011; 70(2): 130-134, indexed in Pubmed: 21630235.

7. Hiatt JR, Gabbay J, Busuttil RW. Surgical anatomy of the hepatic arteries in 1000 cases. Ann Surg. 1994; 220(1): 50-52, indexed in Pubmed: 8024358.
8. Michels NA. Newer anatomy of the liver and its variant blood supply and collateral circulation. Am J Surg. 1966; 112(3): 337-347, indexed in Pubmed: 5917302.

9. Miyaki T. Patterns of arterial supply of the human fetal liver. The significance of the accessory hepatic artery. Acta Anat (Basel). 1989; 136(2): 107-111, indexed in Pubmed: 2816258.

10. Mlakar B, Gadzijev EM, Ravnik D, et al. Anatomical variations of the cystic artery. Eur J Morphol. 2003; 41(1): 31-34, doi: 10.1076/ejom.41.1.31.28103, indexed in Pubmed: 15121546.

11. Polguj $M$, Podgórski $M$, Hogendorf $P$, et al. Variations of the hepatobiliary vasculature including coexistence of accessory right hepatic artery with unusually arising double cystic arteries: case report and literature review. Anat Sci Int. 2014; 89(3): 195-198, doi: 10.1007/s12565-013-02195, indexed in Pubmed: 24310410.

12. Shaw BW, Iwatsuki S, Starzl TE. Alternative methods of arterialization of the hepatic graft. Surg Gynecol Obstet. 1984; 159(5): 490-493, indexed in Pubmed: 6387980 .

13. Skórzewska A, Stajgis P, Grzymisławska M, et al. Rare variations of hepatic arteries in association with variable origin of gastroduodenal artery found in multidetector computed tomography angiography. Folia Morphol. 2014; 73(4): 531-535, doi: 10.5603/FM.2014.0082, indexed in Pubmed: 25448917. 